\title{
Live Birth following Single Embryo Transfer derived from Vitrified Oocyte and Surgically Retrieved Frozen Sperm in a Poor Responder Patient
}

\author{
Mandeep Kaur, Manishi Mittal, Anuja Kamath, K Deepika, Anu Kottur, Kamini A Rao
}

\begin{abstract}
We report a live birth following transfer of a single embryo derived from vitrified and thawed oocyte in a 30-year-old poor responder patient. Intracytoplasmic sperm injection was done with frozen thawed sperm obtained surgically, as the male partner was suffering from anejaculation and was a poor candidate for other non-surgical methods in view of his medical comorbidities.
\end{abstract}

Keywords: Vitrification, Slow freezing, Oocyte surgical retrieval of sperms, Frozen sperms, Vitrified oocyte.

How to cite this article: Kaur M, Mittal M, Kamath $A$, Deepika K, Kottur A, Rao KA. Live Birth following Single Embryo Transfer derived from Vitrified Oocyte and Surgically Retrieved Frozen Sperm in a Poor Responder Patient. Int J Infertility Fetal Med 2013;4(2):62-65.

\section{Source of support: Nil}

Conflict of interest: None

\section{INTRODUCTION}

Oocyte cryopreservation is a technique recently gaining much attention and is an important part of assisted reproduction. Vitrification or rapid freezing of oocyte has a good outcome as it avoids the formation of intra and extracellular ice crystals. It is simple and does not require programmable freezing equipment.

Oocyte in its second metaphase is frozen at an extremely rapid rate (around $20,000^{\circ} \mathrm{C}$ per minute), therefore, leads to minimal chance of ice crystal formation. This is achieved by exposing the oocytes to a higher concentration of cryo-protective agents. We report this in a poor responder patient, wherein the oocytes were vitrified in an emergency situation due to nonavailability of sperms, and later after thawing were fertilized with frozen thawed surgically retrieved sperms. The procedure resulted in a single embryo which was transferred and resulted in a live birth at our center.

\section{CASE REPORT}

Mrs N, a 30-year-old female, reported on 16/3/12 with primary infertility for 9 years. She was diagnosed to have a

Date of Received: 08-08-13

Date of Acceptance: 18-08-13

Date of Publication: May 2013 deranged glucose tolerance test and subclinical hypothyroidism 1 year back and was being treated for it. Her AMH was $0.8 \mathrm{ng} / \mathrm{ml}$ and AFC was 8, indicating a probable poor response during controlled ovarian stimulation.

Sexual history of the couple was normal in the past but, over the last 2 to 3 years, the husband developed anejaculation. He was 38 years old, diagnosed to have type-2 diabetes mellitus 4 years back and was prescribed oral hypoglycemics. He was also diagnosed to have dilated cardiomyopathy with history of septal myocardial infarction. His echocardiography report showed an ejection fraction of $36 \%$ with mild mitral and tricuspid regurgitation.

The couple was counseled for IVF-intracytoplasmic sperm injection (ICSI) with percutaneous epididymal sperm aspiration (PESA) in view of long duration of infertility. The husband underwent fitness for anesthesia. In view of medical history, he was advised to continue all the medicines except clopidogrel which he had to stop 3 days before the procedure.

In view of expectation of poor response, controlled ovarian stimulation was started with antagonist protocol using recombinant gonadotropins which were started from 2nd day of her cycle. Injection cetrorelix $(0.25 \mathrm{mg})$ was started on day 6 of the stimulation till the day of trigger. On day 10 of the stimulation, there were three follicles in the right ovary (1 dominant and 2 intermediate) and three in the left (all intermediate). The couple was explained about this mono-follicular response, but they elected to continue the treatment. The trigger was given with recombinant HCG (ovitrelle $250 \mu \mathrm{g}$ ) on day 11 of stimulation.

Oocyte retrieval was done on day 13 of the stimulation; double lumen needle was used and four oocytes (3 mature) were obtained. PESA was planned on the same day, but it was found that the husband had forgotten to stop clopidogrel. In view of risk factors, the procedure was abandoned and the three oocytes were vitrified.

Vitrification was done 2 hours after the retrieval, after ensuring maturity by denuding cumulus cells. Oocytes were suspended for 10 minutes in an equilibration solution (SAGE media) at room temperature, and then passed through a droplet of vitrification solution for 30 seconds. Then oocytes 

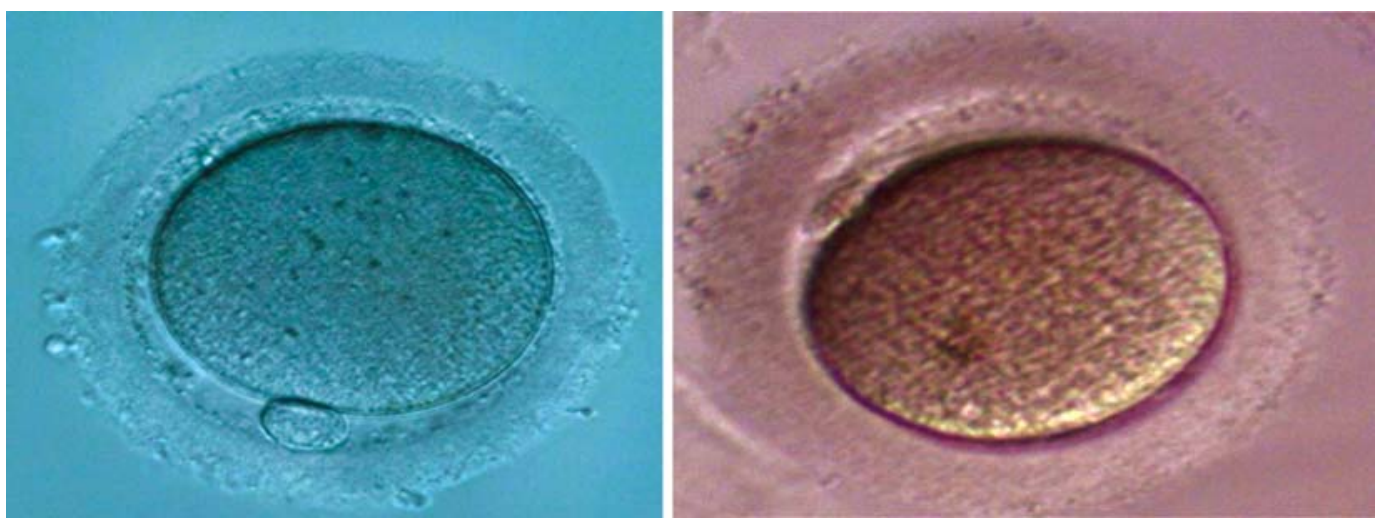

Fig. 1: Oocytes after thawing and before ICSI

were loaded into the cryolock and quickly plunged into liquid nitrogen.

At a later date, PESA was tried but since no sperms were obtained, TESA (testicular sperm aspiration) was done and the sperms were frozen.

The patient reported after 2 months for embryo transfer. Endometrium was developed with estrogen (estradiol valerate $2 \mathrm{mg}$ ). When the endometrial thickness became $9.6 \mathrm{~mm}$ (triple line pattern), injectable progesterone $50 \mathrm{mg}$ intramuscular once a day was started. Same day the frozen oocytes and sperms were thawed and ICSI was done.

Warming of oocytes started with the removal of cryolock from liquid nitrogen dewar and immersing in warming solution containing decreasing concentration of sucrose (1 M for $1 \mathrm{~min}, 0.5 \mathrm{M}$ in 2 drops 2 mins each) (1 min in $1 \mathrm{M}$, 2 mins each in 2 drops of $0.5 \mathrm{M}$ ) and then immersed into MOPS (3 drops for 3 minute each). Oocytes were left in media for 3 hours before ICSI was performed. Three mature oocytes were thawed out of which only two survived (Fig. 1) All recovered oocytes which have good survival should have intact zona, clear perivitelline space, clear cytoplasm and intact polar body. ICSI was done and injected oocytes were placed in bench top incubator with triple gas (K systems).

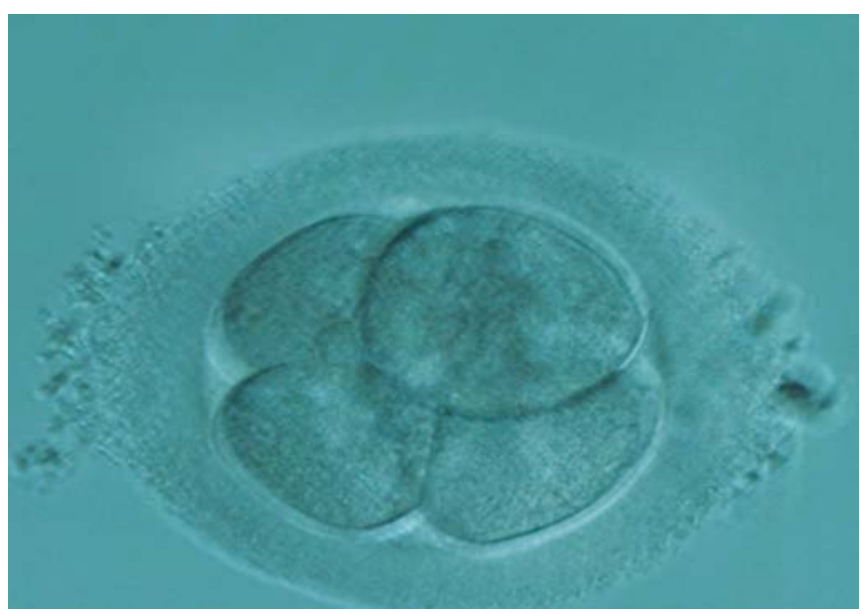

Fig. 2: One four-cell embryo that was transferred
Fertilization check was done after 18 hours and only one injected oocyte had fertilized and started cleaving and reached four-cell stage on day 2 (Fig. 2). In view of a single embryo patient was called for transfer on day 2. Embryo transfer was done on day 2 with soft catheter using afterloading technique.

Luteal phase support was given in form of estradiol valerate (2 mg three times a day), natural micronized progesterone (400 mg vaginally twice a day), dydrogesterone $10 \mathrm{mg}$ twice a day), HCG 5000 IU for three doses (at interval of 5 days each). Peri-implantation steroid (dexamethasone $0.5 \mathrm{mg}$ once a day) and low molecular weight heparin (Enoxaparin $20 \mathrm{mg}$ daily) were also given for 5 days.

At 4-week 1 day, $\beta$-HCG was 2105 IU/l and gestational sac $(0.46 \mathrm{~cm})$ was visualized on scan. She had an episode of bleeding for which she was admitted at 4-week 6 days and managed conservatively. Cardiac activity was observed at 6 weeks 4 days. Estrogen and progesterone were tapered at around 12 weeks. NT and double marker showed low risk (13-week 6 days). Anomaly scan (18-week) and fetal echo were also found to be normal. She developed GDM at 23 weeks and was put on insulin. Prophylactic steroids were given at 31 weeks and, in view of all, the risk factors elective LSCS was planned at 38 weeks.

Elective LSCS was done under spinal anesthesia and a live-term female baby was extracted as vertex, birth weight $3.3 \mathrm{~kg}$ and apgar score of 8/10 and 9/10.

\section{DISCUSSION}

Cryopreservation refers to cooling of cells and tissues to sub-zero temperature in order to stop biological activity and preserve them for future use. First human birth from frozen sperm was reported in 1953. ${ }^{1}$ In 1940s, it was found that glycerol could protect sperm from damage during cryopreservation and thawing. In the 1970s, other cryoprotectants like propanediol, ethylene glycol and dimethyl 
sulfoxide (DMSO) were identified and found to minimize cell damage. Slow freezing techniques were developed with programmable freezers to permit cell dehydration to prevent ice-crystal formation.

These improvements led to birth of first human birth from frozen embryo in $1984^{2}$ and frozen oocyte in 1986. ${ }^{3}$ Over the last few years, vitrification has developed as a new upcoming technique. Vitrification of oocytes is no more an experimental procedure. Vitrification is a technique using initial high concentration of cryoprotectant and ultrarapid cooling to solidify the cell in a glass-like state without formation of ice. Mature oocyte cryopreservation was less successful as compared to embryos due to its large size, water content and chromosomal arrangement. The meiotic spindle is most labile to damage during freezing and thawing and these abnormalities are dependent on age of the patient and the technique that has been used. ${ }^{4}$ Modifications in technique have increased survival rates. ${ }^{5}$

Most vitrification systems are open and involve direct exposure of the gametes to liquid nitrogen. There is little concern of the potential exposure to infectious organisms. As of September 2012, open systems are not FDA approved, but, whether closed systems are equally effective, is not so clear. However, no cases of exposure associated infection transmission have been reported and methods are being devised to sterilize the liquid nitrogen. ${ }^{6}$

Cryopreservation protocols involve removal of the cumulus cells. Removal of cumulus cells decreases chance of fertilization following standard insemination, and zona hardening after thawing cryopreserved oocytes makes ICSI the preferred mode of fertilization. But, it is not clear that ICSI is necessary for fertilization of frozen thawed oocytes. ${ }^{7}$ In a randomized control trial by Cobo et al, ${ }^{5}$ it is suggested that fertilization and clinical pregnancy rates were similar when fresh and frozen oocytes were compared in young patients. Yet, it is not clear that this data can be generalized, particularly in older women and different laboratories may be using different protocols and having different success rates.

Observational studies done in Italy where supernumerary oocytes were frozen, suggested that implantation and pregnancy rates were lower when frozen oocytes were compared to fresh oocytes or frozen embryos. ${ }^{8}$ Data is limited but it has been demonstrated that success rates are more in young and highly selected group of patients. Clinic specific rates should be used to counsel the patients. No major congenital malformations have been seen in children born by this technique; ${ }^{9}$ however long-term studies still need to be done before a final conclusion can be made.
Some of the indications for oocyte vitrification are in donor cycles where synchronization between donor and recipient is not required but till more reassuring data is available, universal oocyte banking is not advocated. Mature oocyte banking can be done if partner is not available or freezing embryos are not considered due to religious reasons and in patients of chemotherapy, radiotherapy or oophorectomy for other indications.

Failure to obtain sperm for IVF is another important indication. Two studies have been reported in literature with this indication. However, the number of oocyte in these studies was not as low as is reported in our study. ${ }^{10,11}$ Oocyte cryopreservation is also an option for couples who wish not to cryopreserve embryos that are not transferred in fresh cycles and it will add on to the cumulative pregnancy rates.

Elective cryopreservation to defer childbearing is another indication but data is insufficient to recommend freezing for this indication. Success rates are very low for patients who cryopreserve at the age more than 38 years of age and it gives a false sense of security. All patients should be counseled with clinic specific success rates, age, risk, cost and alternatives to this approach.

\section{CONCLUSION}

Vitrification of oocytes is an upcoming technique as it is not so commonly being used in our clinical practice. High concentration of cryoprotectant and rapidity of the procedure decreases the chance of intracellular and extracellular ice formation. Vitrification of the oocyte gives better success rates than previously used slow freezing protocols. This is a first case in India with the use of frozen oocyte and frozen sperm resulting in term uncomplicated live birth following single embryo transfer.

\section{REFERENCES}

1. Sherman J. Synopsis of the use of human semen since 1964: The state of art of semen banking. Fert Stert 1973;24:397-412.

2. First baby born of frozen embryo. New York Times 1984.

3. Chen C. Pregnancy after human oocyte cryopreservation. Lancet 1986;1:884-886.

4. Baka SG, Toth TL, Veeck LL, Jones HW, Muasher SJ, Lanzendorf SE. Evaluation of the spindle apparatus in in vitro matured human oocytes following cryopreservation. Hum Reprod 1995;10:1816-20.

5. Cobo A, Diaz C. Clinical application of oocyte vitrification: A systematic review and met analysis of randomized controlled trials. Fertil Steril 2011;96:277-285.

6. Parmegiani L, Cognigni G, Bemardi S, Cuomo S, Ciampaglia $\mathrm{W}$, Infante FE, et al. Efficiency of aseptic open vitrification and hermetical cryostorage of oocytes. Reproductive Biomedicine Online 2011;23:505-512.

7. Fabbri R, Porcu E, Marcella T, Primavera MR, Seracchioli R, Ciotti PM, et al. Oocyte Cryopreservation 1998;13(suppl 4): 98-108. 
8. Borini A, Levi Setti PE, Anserini P, De Luca R, De Santis L, Porcu E, et al. Multi-center observational study on slow-cooling oocyte cryopreservation: Clinical Outcome. Fertil Steril 2010;94:1662-1668.

9. Noyes N, Porcu E, Borini A. Over 900 oocyte cryopreservation babies born with no apparent increase in congenital anomalies. Reprod Biomed Online 2009;18:769-776.

10. Song WY, Sun YP, Jin HX, Xin ZM, Su YC, Chian RC. Clinical outcome of emergency egg vitrification for women when sperm extraction from the testicular tissues of the male partner is not successful. Syst Biol Reprod Med 2011;57:210-213.

11. Virant-Klun I, Bacer-Kermavner L, Tomazevic T, VrtacnikBokal E. Slow oocyte freezing and thawing in couples with no sperm or an insufficient number of sperms on the day of in vitro fertilization. Reprod Biol Endocrinol 2011;9:19.

\section{ABOUT THE AUTHORS}

\section{Mandeep Kaur (Corresponding Author)}

Fellow, Department of Reproductive Medicine, Bangalore Assisted Conception Centre, Bengaluru, Karnataka, India, Phone: 08884838538 e-mail: docmandeep80@gmail.com

\section{Manishi Mittal}

Fellow, Department of Reproductive Medicine, Bangalore Assisted Conception Centre, Bengaluru, Karnataka, India

\section{Anuja Kamath}

Senior Embryologist, Department of Reproductive Medicine Bangalore Assisted Conception Centre, Bengaluru, Karnataka, India

\section{K Deepika}

Consultant, Department of Reproductive Medicine, Bangalore Assisted Conception Centre, Bengaluru, Karnataka, India

\section{Anu Kottur}

Consultant, Department of Reproductive Medicine, Bangalore Assisted Conception Centre, Bengaluru, Karnataka, India

\section{Kamini A Rao}

Medical Director, Department of Reproductive Medicine, Bangalore Assisted Conception Centre, Bengaluru, Karnataka, India 